\title{
Estimation of I-131 Concentration Using Time History of Pulse Height Distribution at Monitoring Post and Detector Response for Radionuclide in Plume
}

\author{
Hideo HIRAYAMA ${ }^{1, *}$, Masatsugu KAWASAKI ${ }^{2}$, Hiroshi MATSUMURA ${ }^{1}$, \\ Takehisa OHKURA ${ }^{2}$, Yoshihito NAMITO ${ }^{1}$, Toshiya SANAMI ${ }^{1}$, \\ Mitsumasa TAKI $^{2}$, Tetsuya OISHI $^{2}$ and Michio YOSHIZAWA ${ }^{2}$ \\ ${ }^{1}$ High Energy Accelerator Research Organization, 1-1 Oho, Tsukuba-shi, Ibaraki 305-0801, Japan \\ ${ }^{2}$ Nuclear Science Research Institute, Japan Atomic Energy Agency, 2-4 Shirane, Shirakata, Tokai-mura, Naka-gun, \\ Ibaraki 319-1195, Japan
}

\begin{abstract}
A method of deducing the $\mathrm{I}-131$ concentration in a radioactive plume from the time history of peak count rates determined from pulse height spectra obtained from an $\mathrm{NaI}(\mathrm{Tl})$ scintillation detector employed as a detector of a monitoring post was presented. The contribution to the count rates from I-131 accumulated around the monitoring post was subtracted in accordance with the time history, taking into consideration the cumulative attachment and its decay. The concentrations of I-131 in the plumes were estimated from the count rates using the calculated response of the $\mathrm{NaI}(\mathrm{Tl})$ detector with egs 5 for a model of a plume uniformly containing I-131. This method was applied to the data from the monitoring posts at Nuclear Science Research Institutes of Japan Atomic Energy Agency (JAEA). The estimated time history variation of I-131 concentrations in plumes was in fair agreement with those measured directly by an air sampling method. The difference was less than a factor of 4 for plumes that arrived on March 15 and March 21, indicating relatively high I-131 concentrations among the plumes studied in this work.
\end{abstract}

KEYWORDS: iodine 131, plume, time history, Monte Carlo, egs5, Nal scintillator

\section{Introduction}

The radionuclide concentrations in plumes dispersed over a wide area resulting from the accident that occurred at the Fukushima Daiichi Nuclear Power Plant (hereinafter referred to as the "plume") are important in evaluating the internal exposure dose via inhalation. In particular, the concentration of I-131 is important from the perspective of radiation-induced thyroid cancer risk assessment. Hirayama et al. attempted to estimate the concentrations of radionuclides contained in the plumes that arrived using the pulse height distribution measured on expressways with a $\phi 1.5^{\prime \prime} \times 1.5^{\prime \prime} \mathrm{LaBr}_{3}$ scintillation spectrometer ${ }^{1)}$. Because the measurements on

\footnotetext{
* Corresponding author, E-mail: hideo.hirayama@kek.jp

DOI : 10.15669/fukushimainsights. Vol.4.295

(C) 2021 Atomic Energy Society of Japan. All rights reserved.

Originally published in Transactions of the Atomic Energy Society of Japan (ISSN 1347-2879), Vol. 13, No. 3, p.119-126

(2014) in Japanese. (Japanese version accepted: July 2, 2014)
} 
expressways were not continuously performed at the same location, it was difficult to deduce the contribution of I-131 deposited on the ground surface. In this study, we propose a method to estimate the contribution of radionuclides deposited on the soil surface ${ }^{\text {a }}$ resulting from the arrival of the plume, separately from the contribution of radionuclides contained in the plume, from the time histories of peak count rates obtained from the pulse height distribution at monitoring posts.

To validate this method, it was applied to monitoring posts placed in the area around the perimeter monitoring zone set up by the Nuclear Science Research Institute (NSRI) of the Japan Atomic Energy Agency (JAEA) as well as in Tokai Village (hereinafter referred to as "the village"). We estimate the time history of I-131 in the air using the pulse height distribution data measured at the monitoring posts to compare it to the time history of the concentration measured by sampling the air in the NSRI premises ${ }^{2)}$.

The peak count rates for I-131 in the plume were converted to I-131 concentrations using the peak detection efficiency per $\mathrm{Bq} / \mathrm{cm}^{3}$ of $365 \mathrm{keV} \gamma$ ray of $\mathrm{I}-131$, which was obtained by applying the method to replace a photon flux from a plane isotropic source with a point isotropic source and plane detector ${ }^{3)}$ to the egs $5^{4)}$ electromagnetic cascade Monte Carlo code, as described in reference 1 .

\section{Values Measured at Monitoring Posts}

\section{Layout of Monitoring Posts}

NSRI has established monitoring posts at 14 locations in the perimeter monitoring zone and the village as shown in Firure 1 to perform continuous measurement of the air absorbed dose rate. As a detector, a $\phi 2^{\prime \prime} \times 2^{\prime \prime} \mathrm{NaI}(\mathrm{Tl})$ scintillator, which was partially surrounded by a 7-mmthick lead shield to improve the photon energy characteristics to the air absorbed dose rate, was placed at a height of $2.7 \mathrm{~m}$ above the ground surface. Among the monitoring posts, the measurement results obtained at MP-11, MP-21, MP-22, MP-24, and MP-25 were used for analysis.

The conditions of the areas surrounding the respective monitoring posts were as follows. MP11 is on the western boundary near the north gate of the NSRI. There are roads on the premises and pine trees along its southern and northern sides. MP-21 is on the premises of the elementary school of the village (at the time of placement; it is currently used as a schoolchildren's club), and is surrounded by several types of trees. At the south side is a dry field. MP-22 is on a street in an industrial complex in the village and faces a road and a slope on its northeast and southwest sides, respectively. MP-24 is in a residential district and faces a road and houses on its north and south sides, respectively. MP-25 is in a dry field, and at its southeast side is a descending slope.

\section{Time History of Air Absorbed Dose Rate and I-131 Peak Count Rate}

For each monitoring post, we determined the net count rate corresponding to the total absorption peak of $365 \mathrm{keV}$ of I-131 using the pulse height distribution data of the $\mathrm{NaI}(\mathrm{Tl})$ detector measured every $10 \mathrm{~min}$ and adopted this value as the peak count rate. Figures $\mathbf{2}$ and $\mathbf{3}$ show the time history of the air absorbed dose rate (10-min average) from March 15 to March 24 and the time history of the peak count rate, respectively. These figures show that the time history of

\footnotetext{
a Here, "radionuclides deposited on the soil surface" represent the radionuclides which exist not only on the soil surface but also at monitoring posts and surrounding areas, but excluding the ones contained in the plume.
} 


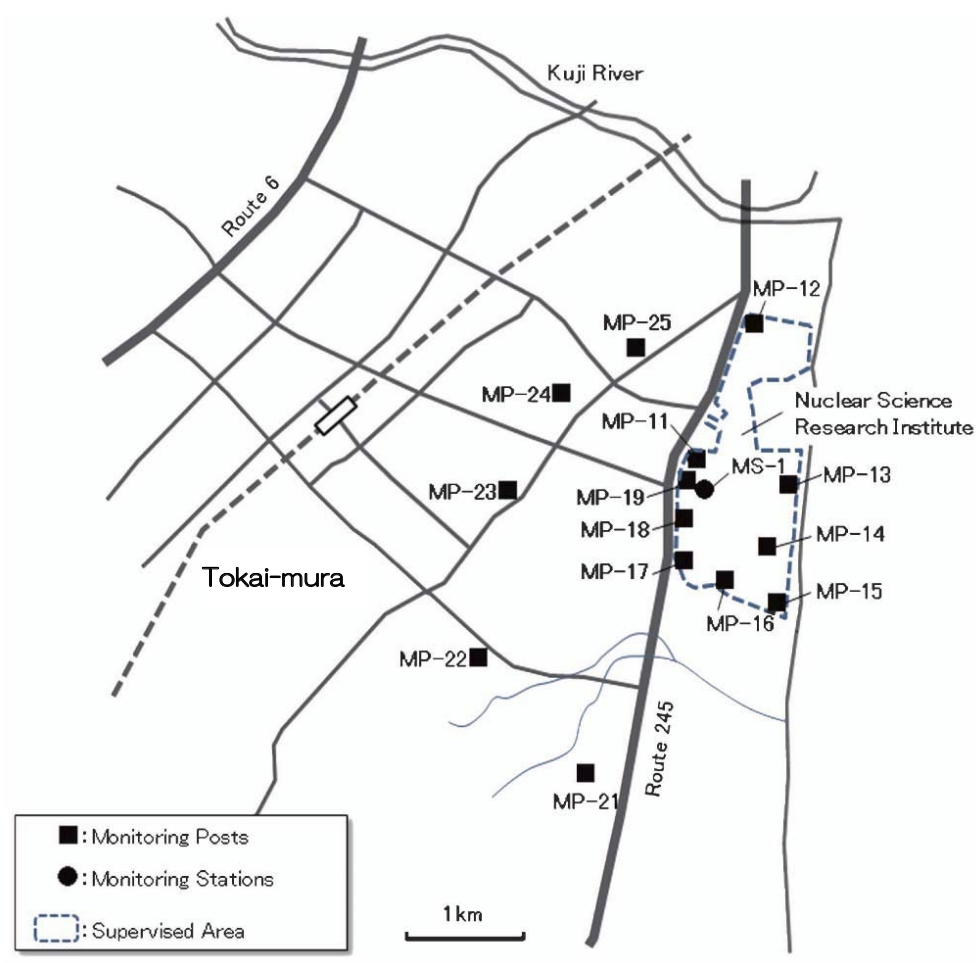

Figure 1 Map of monitoring posts at Nuclear Science Research Institutes of JAEA

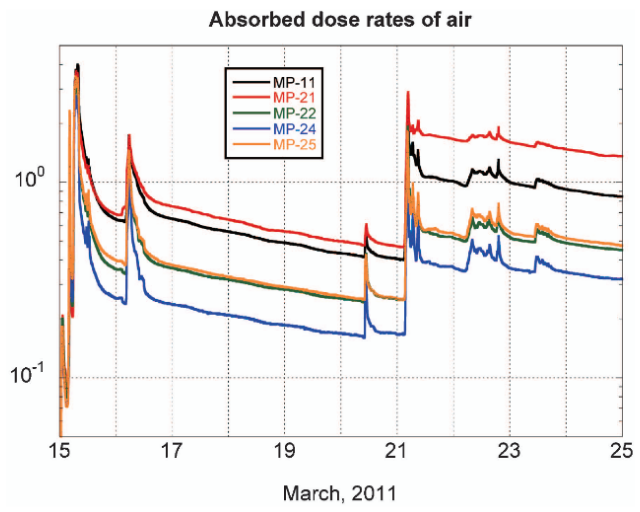

Figure 2 Time variation in absorbed dose rates of air from March 15 to 24 in 2011

the I-131 peak count rate is different from the time history of its air absorbed dose rate. At all of the monitoring posts, while the I-131 peak count rate showed the most noticeable increase on March 21, the air absorbed dose rate was highest on March 15. This means the compositions of the radionuclides contained in the plumes that arrived differ depending on the plume. In addition, the attenuation trend of the air absorbed dose rate resulting from the radionuclides which were deposited after the plume left is different from the I-131 attenuation trend. Therefore, it was found that the I-131 concentration in the plume cannot be estimated only from the air absorbed dose rate and the ambient dose equivalent rate. 


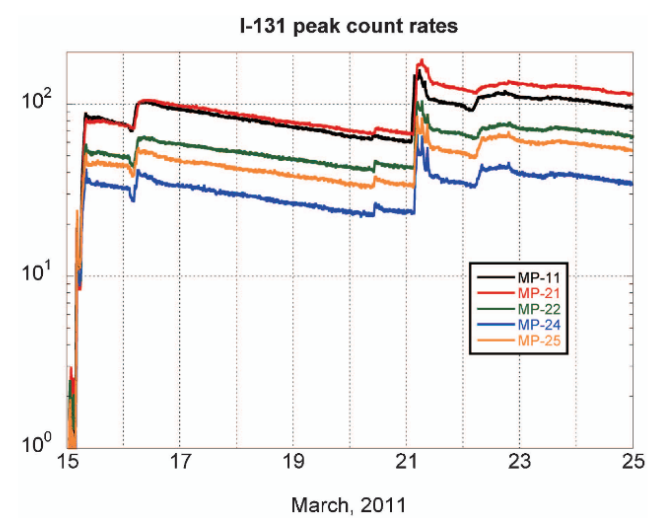

Figure 3 Time variation in I-131 peak count rates from March 15 to 24 in 2011

\section{Estimation of Plume Arrival Time}

Although the plume arrival time and passage completion time can be determined from the time history of the I-131 concentration in the sampled air, we used the method described in the following to determine the arrival time and passage completion time of the locations where the only data available were the data obtained at a monitoring post.

In the time history of the peak count rate corresponding to $365 \mathrm{keV}$ of each monitoring post used for estimating the concentration, the time of measurement when the count rate began to increase was determined as the plume arrival time and the time of measurement when the count rate completed its increase and began to attenuate with a half-life of I-131 was determined as the plume completion time. When it was difficult to determine based on the time history of the I-131 peak count rate, we estimated the time when the plume passed based on the time history of the air absorbed dose rate which changed more significantly. Table 1 shows the plume arrival time and completion time obtained using this method. Because the monitoring posts which were considered this time were located in a small area, the arrival time did not appear to vary significantly among the monitoring posts. The reason why the estimated time, particularly the completion time, varied significantly is because the change in the count rate after the plume passes is relatively small as the deposition increases and uncertainty remains over the determination of the time when the plume has passed. The start and stop times shown in Table 1 are a result of the uncertainty of the process used to determine the arrival time based on this method.

\section{Estimation of the Contribution of the Deposited Radionuclides Based on the Time History of the Peak Count Rates of Pulse Height Distribution}

At the monitoring posts, measurements were repeatedly performed for a predetermined measurement time (measurement section). The average count rate (cps) calculated from the peak counts corresponding to the radionuclides considered within the measurement time was used for the analysis.

For the plumes that arrived, the variables are defined as follows:

$D_{0}$ : The count rate in the measurement section immediately before a plume arrives, in cps

(the count rate corresponding to the contribution of the radionuclides deposited before 
Table 1 Start and stop time used for analysis at each plume and monitoring post

\begin{tabular}{|c|c|c|c|c|c|}
\hline & MP-11 & MP-21 & MP-22 & MP-24 & MP-25 \\
\hline & \multicolumn{5}{|c|}{ March 15} \\
\hline Plume start & $0: 54$ & $0: 47$ & $0: 48$ & $0: 41$ & $0: 41$ \\
\hline Plume stop & $3: 34$ & $3: 27$ & $3: 38$ & $2: 21$ & $2: 21$ \\
\hline Plume start & $3: 34$ & $3: 27$ & $3: 38$ & $3: 41$ & $2: 21$ \\
\hline Plume stop & $5: 34$ & $4: 57$ & $5: 28$ & $5: 41$ & $3: 21$ \\
\hline Plume start & $5: 44$ & $5: 37$ & $5: 58$ & $5: 51$ & $3: 31$ \\
\hline \multirow[t]{2}{*}{ Plume stop } & 11:14 & $9: 57$ & $10: 38$ & $8: 51$ & $9: 21$ \\
\hline & \multicolumn{5}{|c|}{ March 16} \\
\hline Plume start & $4: 16$ & 4:07 & $4: 28$ & $4: 21$ & $3: 51$ \\
\hline \multirow[t]{2}{*}{ Plume stop } & 10:06 & $8: 37$ & $11: 28$ & $12: 51$ & $9: 31$ \\
\hline & \multicolumn{5}{|c|}{ March 20} \\
\hline Plume start & $9: 46$ & $9: 37$ & 10:08 & $9: 21$ & 10:11 \\
\hline \multirow[t]{2}{*}{ Plume stop } & $13: 26$ & $13: 37$ & $13: 48$ & $12: 51$ & $13: 51$ \\
\hline & \multicolumn{5}{|c|}{ March 21} \\
\hline Plume start & $2: 46$ & 3:07 & $3: 18$ & $3: 11$ & $2: 51$ \\
\hline \multirow[t]{2}{*}{ Plume stop } & $10: 56$ & 10:27 & $14: 25$ & $10: 51$ & 11:11 \\
\hline & \multicolumn{5}{|c|}{ March 22} \\
\hline Plume start & $5: 16$ & $5: 28$ & 5:06 & $6: 21$ & $5: 11$ \\
\hline \multirow[t]{2}{*}{ Plume stop } & $23: 36$ & $23: 38$ & $21: 26$ & 23:11 & $21: 41$ \\
\hline & \multicolumn{5}{|c|}{ March 23} \\
\hline Plume start & $10: 26$ & 11:08 & 10:46 & $10: 31$ & $9: 51$ \\
\hline Plume stop & $14: 56$ & $20: 58$ & $16: 36$ & $14: 51$ & $17: 41$ \\
\hline
\end{tabular}

a plume arrives)

$D_{1}$ : The count rate after a plume passes, in cps (the count rate including the contribution of the radionuclides deposited when the plume passes)

$\lambda$ : The decay constant of the radionuclide $(1 / \mathrm{min})$

$t_{c}:$ Measurement time (min)

$i$ : The measurement number in the plume

$m$ : The number of measurements conducted while the plume is arriving

$n_{i}:$ The count rate in the case of the measurement number $i$, in cps

$p_{i}$ : The count rate based on the radionuclide in the plume in the case of the measurement number $i$, in cps

$d_{i}$ : The increment of the count rate based on the radionuclide deposited in the case of the measurement number $i$, in cps

$n_{i}^{\prime}:$ The count rate in the case of the measurement number $i$ from which the count rate of the contribution of the radionuclides deposited before the plume arrives taking into account the attenuation from the time of plume arrival, in cps

$$
\begin{aligned}
& n_{i}^{\prime}=n_{i}-D_{0} \times \exp \left(-(i-1) \times t_{c} \times \lambda\right) \\
& n_{i}^{\prime}=0.0
\end{aligned}
$$

$n_{m}{ }^{\prime}$ : The count rate based on the radionuclides deposited which increased as a result of the passage of the relevant plume, in cps

$$
n_{m}{ }^{\prime}=D_{1}-D_{0} \times \exp \left(-(m-1) \times t_{c} \times \lambda\right)
$$

We assume that a new deposition proportional to the count rate obtained by deducting the contribution of the radionuclides deposited before the relevant measurement section from $n_{i}{ }^{\prime}$ 
(The count rate based on the radionuclides in the plume in the measurement section) was generated as a result of the passage of the plume in each measurement section, and that the count rate obtained as a result of the deposition in the next measurement section accordingly increased. For the contribution of the radionuclides deposited, the attenuation after deposition was considered. The proportionality coefficient of the depositional rate was assumed to be constant while a plume was arriving, and the initial value was set to $f_{0}=0.1^{\mathrm{b}}$.

While applying the aforementioned assumption to the actual measurement values, the calculation was repeated until the relative error between the count rate based on the radionuclides deposited immediately after the plume passed and $n_{m}{ }^{\prime}$ became $1 \%$ or less with the proportionality coefficient which uses the ratio of both values as a correction. The count rate based on the radionuclides contained in the plume in each measurement section was obtained using $d_{i}$ in cases where the relative error was $1 \%$ or less.

During the first phase, the increment $d_{i}$ of the count rate based on the depositional amount increased in each measurement section because of the passage of the plume, using $f_{0}$.

$d_{i}$ does not become negative. Therefore, when $d_{i}$ becomes negative, it should be regarded as zero.

$$
\begin{aligned}
& d_{1}=0.0 \\
& d_{2}=n_{1}{ }^{\prime} \times f_{0}=0.0 \\
& d_{3}=n_{2}{ }^{\prime} \times f_{0} \\
& d_{4}=\left(n_{3}{ }^{\prime}-d_{3}\right) \times f_{0} \\
& d_{5}=\left[n_{4}{ }^{\prime}-\left(d_{3} \times \exp \left(-t_{c} \times \lambda\right)+d_{4}\right)\right] \times f_{0} \\
& \cdots \cdots \\
& d_{m}=\left[n_{m-1}{ }^{\prime}-\sum_{k=3}^{m-1}\left(d_{k} \times \exp \left(-(m-1-k) \times t_{c} \times \lambda\right)\right)\right] \times f_{0} \quad(m \geq 3) \\
& d_{\text {total }, 1}=\sum_{k=3}^{m}\left(d_{k} \times \exp \left(-(m-k) \times t_{c} \times \lambda\right)\right)(m \geq 3)
\end{aligned}
$$

Compare the aforementioned to $n_{m}{ }^{\prime}$, and when

$$
\frac{\left|d_{\text {total }, 1}-n_{m}{ }^{\prime}\right|}{n_{m}{ }^{\prime}}>0.01
$$

$f_{1}=f_{0} \times n_{m}{ }^{\prime} / d_{\text {total }, 1}$ as the new proportionality coefficient and repeat the same operation.

If $d_{\text {total, }, j}$ which satisfies the condition of $\left|d_{\text {total }, j}-n_{m}{ }^{\prime}\right| / n_{m}{ }^{\prime}<0.01$ is obtained, the value $d_{i}$ should be regarded as an increment of the count rate based on the deposition in the case of the measurement number $i$.

Next, the count in each measurement section based on the radionuclides contained in the relevant plume was determined using $d_{i}$. The value $f_{a}$, which is the ratio of the sum of the counts obtained $T_{p}$ to the count rate based on the depositional increase as a result of the passage of the relevant plume $n_{m}{ }^{\prime}$, is a parameter that shows the difference in depositional conditions because of the environment around the monitoring posts and the weather conditions at the time of plume arrival.

\footnotetext{
${ }^{\mathrm{b}}$ If the proportionality coefficient is assumed to be 1 , which is the largest value, the repeat count slightly increases but it has limited influence on the result. Because the results of analyzing several examples converged into a value of approximately 0.1 in many cases, 0.1 was adopted as the initial value.
} 


$$
\begin{aligned}
& T_{p}=n_{1}{ }^{\prime}(=0.0)+\left(n_{2}{ }^{\prime}-d_{1}\right)+\left(n_{3}{ }^{\prime}-\left(d_{1} \times \exp \left(-t_{c} \times \lambda\right)\right)+d_{2}\right)+\cdots+ \\
& \quad\left(n_{m-1}{ }^{\prime}-\sum_{i=1}^{m-2}\left(d_{i} \times \exp \left(-(m-2-i) \times t_{c} \times \lambda\right)\right)\right)=\sum_{i=1}^{m-1} p_{i} \\
& f_{a}=\frac{n_{m}{ }^{\prime}\left(=D_{1}-D_{0}\right)}{T_{p}}
\end{aligned}
$$

Figure 4 shows the schematic relation between the following equation and $p_{i}$ when the convergence conditions are met in the case of $m=8$.

$$
n_{i}^{\prime}, d_{i}, \sum_{k=3}^{i}\left(d_{k} \times \exp \left(-(i-k) \times t_{c} \times \lambda\right)\right)
$$

$d_{i}$ changes in proportion to $p_{i-1}$, and $p_{i}$ and $d_{i}$ becomes zero when $i=8$, showing $n_{i}^{\prime}$ is equivalent to the following equation:

$$
n_{i}^{\prime}=\sum_{k=3}^{i}\left(d_{k} \times \exp \left(-(i-k) \times t_{c} \times \lambda\right)\right)
$$

\section{Calculation of I-131 Detection Efficiency of a Lead- Shielded NaI(TI) Detector with egs5}

As shown in reference 1, the unscattered photons which enter the detector increase slightly as the plume height increases, but the difference is small between $200 \mathrm{~m}$ and $500 \mathrm{~m}$. The plume height was set to $500 \mathrm{~m}$, considering the distance from the Fukushima Daiichi Nuclear Power Plant to Tokai Village, and I-131 was assumed to be spreading infinitely at a uniform density in a horizontal direction from the ground surface to the plume height. By applying the method to replace the gamma-ray flux from a plane isotropic source with a point isotropic source and plane detector ${ }^{3)}$ to egs $5^{4)}$ as described in reference 1 , the photon spectrum of each angle section enters the detector at a height of $2.7 \mathrm{~m}$ from the ground surface in the air of the NTP state $\left(20^{\circ} \mathrm{C}, 1 \mathrm{~atm}\right)$, assuming that the vertical direction distribution is uniform from the ground surface to $500 \mathrm{~m}$.

The $\mathrm{NaI}(\mathrm{Tl})$ detector is structured to project into the upper part of a monitoring post. The

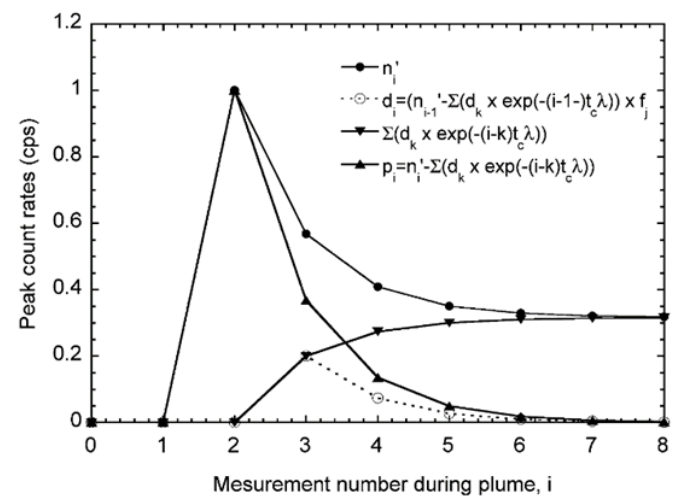

Figure 4 Schematic relation between $n_{i}{ }^{\prime}, d_{i}, \sum_{k=3}^{i}\left(d_{k} \times \exp \left(-(i-k) \times t_{c} \times \lambda\right)\right)$ and $p_{1}$ 
lower structure of the detector and monitoring post contribute to scattered photons but have a limited influence on the non-scattered photons, which may possibly generate a total absorption peak. In the calculation of detection efficiency, considering only the aluminum cover of the $\mathrm{NaI}(\mathrm{Tl})$ detector and lead-shielded $\mathrm{NaI}(\mathrm{Tl})$ detector, the photons having the photon spectra of the respective angle sections previously obtained were assumed to enter the detector at corresponding angles, as the peak detection efficiency corresponding to $365 \mathrm{keV} \gamma$ radiation of I-131, $2.38 \times 10^{4} \mathrm{cps}$ per $\mathrm{Bq} / \mathrm{cm}^{3}$, was obtained. Using this value as a conversion factor, the concentration in the plume was determined from the I-131 peak count rate in the plume obtained according to the method described in Chapter III.

\section{Results and Discussion}

\section{Time History of the Peak Count Rate Caused by the I-131 Contained in the Plumes that Arrived as well as the Deposited I-131}

Figure 5 shows the time history of the peak count rate at MP-21 and MP-24 caused by I-131 contained in the plumes as well as the deposited I-131 during the entire period obtained according to the method described in Chapter III. The peak count rate because of the deposited I-131 was the highest at MP-21 and the lowest at MP-24. Although the difference in the peak count rate resulting from the I-131 contained in the plume was not significant, a difference

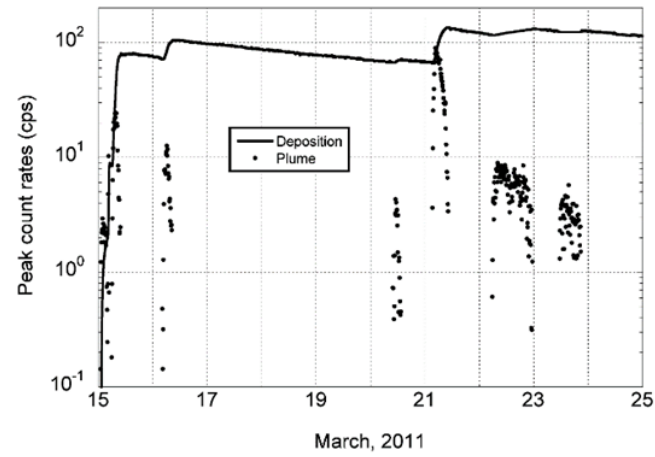

(a) MP-21

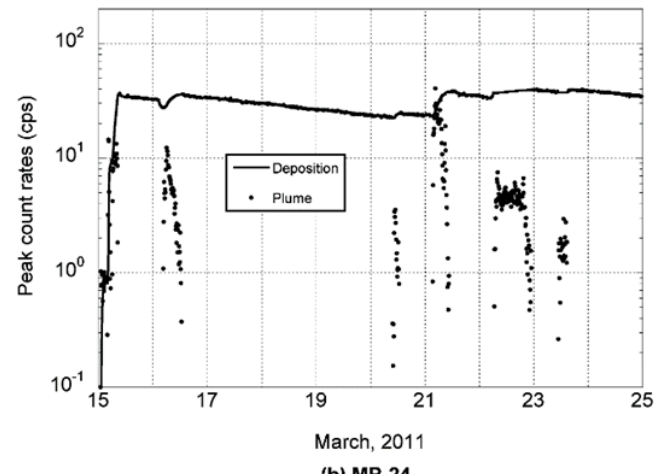

(b) MP-24

Figure 5 Time variation in peak count rates due to I-131 contained in the plume and deposited around (a) MP-21 and (b) MP-24 from March 15 to 24 in 2011 
of nearly 2 or 3 to 1 in the peak count rate resulting from the deposited I-131 was observed between these monitoring posts. This shows that the difference in the environment around the monitoring post makes a difference of from 2 or 3 to 1 in the depositional amount of I-131 and that it is extremely difficult to measure the I-131 concentration contained in the plume from the I-131 depositional density in the way that it has been measured. In both cases, it was found that the plumes that arrived on March 15 and March 21 had a significant influence. A significant decrease in the I-131 peak count rate was observed at MP-24 before the plume arrived on March 16, and the air absorbed dose rate also showed a similar tendency for unknown reasons.

\section{Comparison between the Estimated I-131 Concentration and that Measured using Air Sampling}

Figures 6-8 show the results of comparing the concentrations on March 15, 21, and 22 estimated using the measured pulse height distributions obtained at MP-21 and MP-24 to the measurements obtained by air sampling ${ }^{2}$. The monitoring of the radioactive concentration in the air by air sampling was performed from 01:25 on March 15 to 09:00 on June 6. During the sampling conducted until 7:05 on March 21, a monitoring vehicle was parked in front of MP11 to sample the air using a dust sampler mounted on the vehicle. Subsequent sampling was conducted using a sampler installed at the monitoring station (MS-1).

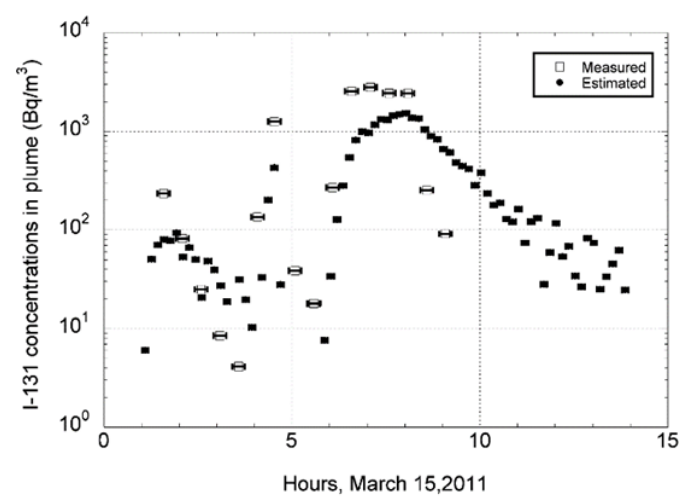

(a) MP-21

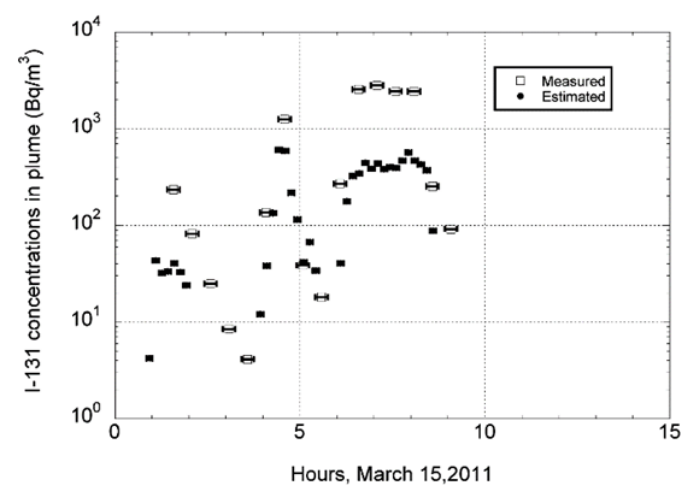

(b) MP-24

Figure 6 Comparison of estimated I-131 concentrations in the plume at (a) MP-21 and (b) MP-24 with those measured by air sampling at March 15, 2011 


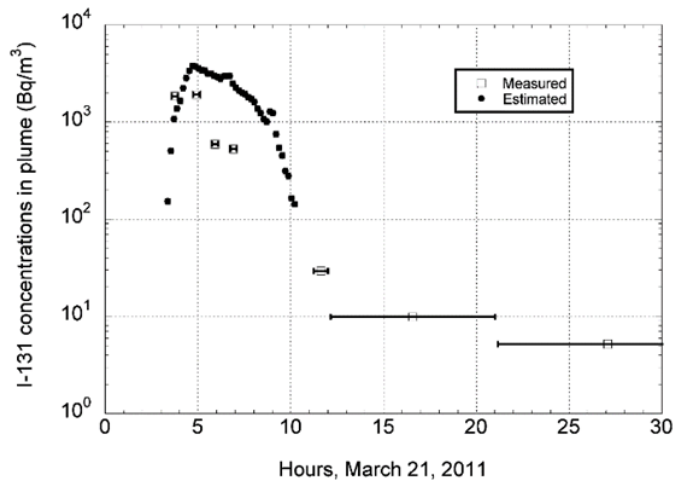

(a) MP-21

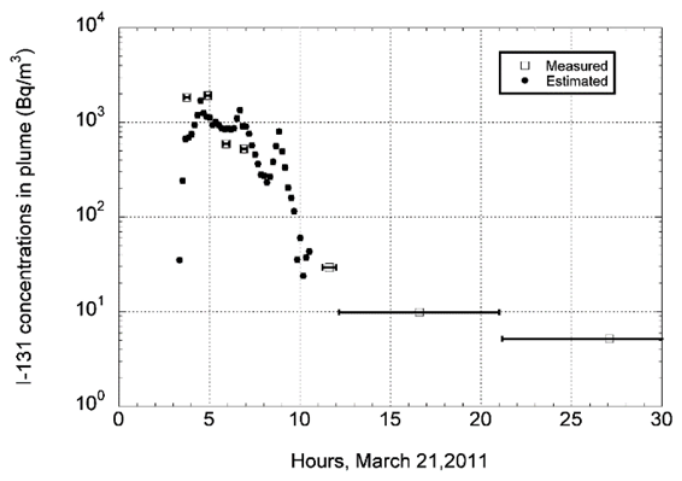

(b) MP-24

Figure 7 Comparison of estimated I-131 concentrations in the plume at (a) MP-21 and (b) MP-24 with those measured by air sampling at March 21, 2011

On March 15, the arrival of a plume was observed for the first time in Tokai Village and the I-131 peak count rate changed very significantly. Concerning the arrival time of the plume, both monitors showed very similar results to those of the air sampling. A significant difference of the time when the plume passed between the results of air sampling and estimated using the measured pulse height distributions was observed at MP-21, reflecting the difficulty in clearly determining the time at which the plume passed. Focusing on the plume at approximately 7:00 when the concentration was highest, the estimated I-131 concentrations at five monitoring posts were underestimated, and were $0.23-0.63$ those of the air sampling measurements.

The results on March 21 correspond to the plumes with the highest I-131 peak count rate. Concerning the time history of the arrival of the plume, both monitors showed results corresponding to the air sampling results. At off-peak times, the estimated concentrations were higher than the measurements at MP-21. At approximately 4:00 when the peak in the measurements was observed, the estimated I-131 concentrations at five monitoring posts were $0.58-1.4$ those of the air sampling measurements.

The results on March 22 were influenced markedly by deposition, and it was difficult to determine the time period of the plume from the time history of the I-131 peak count rate. Although a detailed comparison such as in the case of other plumes could not be performed because the prolonged air sampling time, in terms of the time history of the arrival of the plume there was a good correspondence between the monitoring and air sampling results. However, the estimated I-131 concentrations were consistently higher. The estimated I-131 


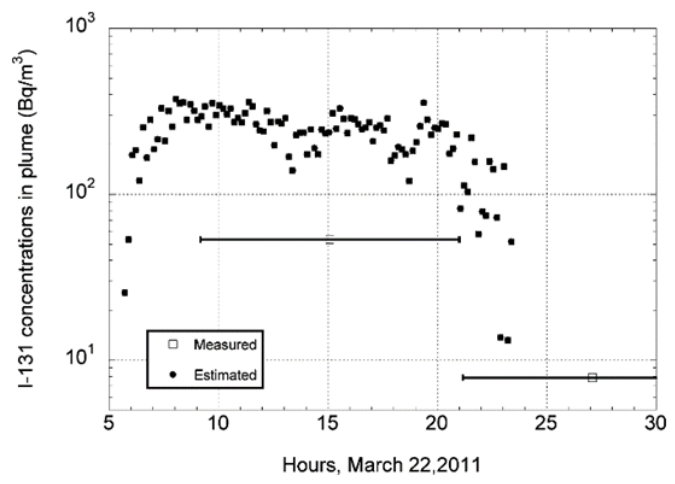

(a) MP-21

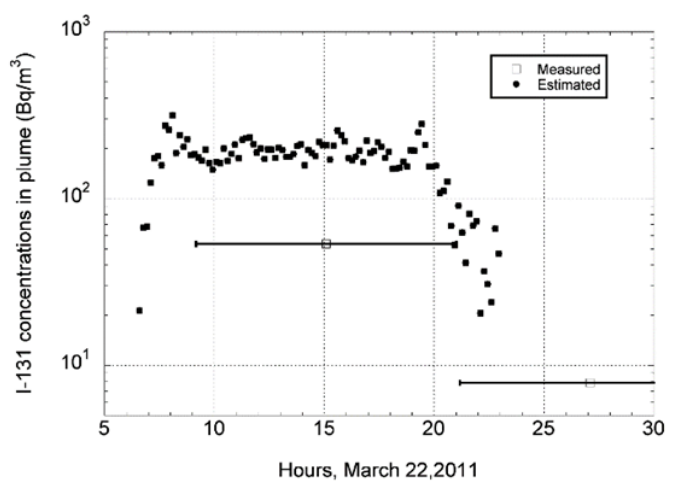

(b) MP-24

Figure 8 Comparison of estimated I-131 concentrations in the plume at (a) MP-21 and (b) MP-24 with those measured by air sampling on March 22, 2011

concentrations at the five monitoring posts were overestimated, and were 3.8-3.9 those of the air sampling measurements.

From the aforementioned results, it can be stated that using this method enables estimation of the time history of the I-131 concentrations in the plume that are near the actual values, although there may be uncertainty in the plume arrival completion time. The extent of the difference from the concentrations obtained through air sampling is up to a factor of 4 in the case of the plume that initially arrived on March 15 as well as the plume containing I-131 at a high concentration such as that which arrived on March 21, corresponding to a factor of 9 in the case where the concentration was low and the plume arrival completion time was difficult to determine. The comparison of the absolute value of the concentrations of sampled air was intended to verify the accuracy of this method in the case where it is used for estimation at a location where no information on the I-131 concentration in the air is available. It was shown that this method can provide much more important information compared to that of the former method in which the time history of the concentration in the air was estimated from the result of calculating an atmospheric dispersion model based on integrated information, the air absorbed dose rate and ambient dose equivalent rate of the radionuclides, which were deposited on the ground surface by the arrival of several plumes, although differing by up to a factor of 9 , which is not insignificant. 


\section{Change in $f_{a}$ Depending on the Time of Plume Arrival}

From the analysis of the change in the peak count rate of I-131 as described in Chapter III, the ratio $f_{a}$ of the total count rate based on I-131 which arrived as a plume to the count rate based on I-131 which was deposited by the plume upon arrival can be determined for each plume.

Regarding the same monitoring post, $f_{a}$ was considered to be a parameter that shows the difference in the depositional conditions caused by weather and the I-131 conditions. In addition, the difference among the monitoring posts in the case of the same plume was regarded as a parameter which depended on deposition condition of each monitoring post due to the difference in surrounding environment. Figure 9 represents $f_{a}$ values of each monitoring post during the observed period. It shows the March 21 to 23 period had smaller $f_{a}$ values than those which arrived on March 15 and 16. While conditions for "dry deposition" occurred on March 15 and 16 because no rainfall was recorded, conditions for "wet deposition" occurred from March 21 to 23 because there was some precipitation. Therefore, this tendency seemed to be in contradiction to "conventional thinking" that the deposition rate was higher in the case of wet deposition. Because the deposition subjected to this analysis included I-131 that adhered to monitoring posts and the areas around them, it is possible that the I-131 which adhered to the surface of the monitoring post near the detector was washed away by the rain and thereby there was a change in the distribution of the I-131 deposited and this had an influence on the readings. In addition, the I-131 conditions such as chemical form varied depending on the time of arrival, which may have had a significant influence on the rate of deposition. To investigate the various factors that affected the relation between the concentration of the radionuclides in a plume and depositional amount, it is necessary to study the change in $f_{a}$ for other nuclides and I-131 observed in other areas.

\section{Conclusions}

We proposed a method for estimating the contribution of radionuclides deposited on the ground surface resulting from plume arrival, separately from the contribution of radionuclides contained in the plume, from the time history of the peak count rates of the radionuclides obtained from the data of the pulse height distributions. We applied this method to five monitoring posts placed in an area around the perimeter monitoring zone of the NSRI to estimate the time history of the I-131 concentrations contained in the plumes that arrived from March

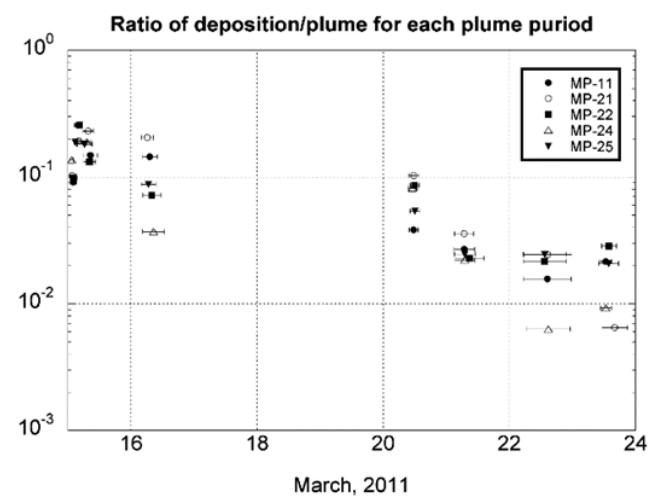

Figure 9 Variation in $f_{a}$ between the plumes and the monitoring posts 
15 to 24,2011 . The time history of the I-131 concentrations obtained showed generally good correspondence to the time history measured by air sampling. Concerning the absolute values of the concentrations, although they varied depending on the ratio of the peak count rate based on the deposited I-131 to the peak count rate based on the I-131 contained in the plume, it was found to be estimated within a range of up to a factor of 4 in the case of the plumes that arrived initially or those containing I-131 at a high concentration as well as within the range of up to a factor of 9 if the concentration was low and the plume completion time was difficult to determine. At the same time, it was also found that the rate of I-131 deposition in the surrounding area varied depending on the conditions around the monitoring posts and the arrival time.

The method we proposed is new and useful in that the time history of the I-131 concentration in the plume, which could not be previously measured without using the air sampling method, can be estimated from the pulse height distribution of the monitoring posts although the accuracy is limited. In the case of the accident that occurred at the Fukushima Daiichi Nuclear Power Plant, the time history of the I-131 concentration in the plumes was not measured in Fukushima Prefecture immediately after the accident. Therefore, the time history of the I-131 concentration in the air was estimated from an atmospheric dispersion model based on dose rate information such as the air absorbed dose rate and ambient dose equivalent rate, data on the density of I-131 deposited on the ground, and data on the density of I-131 estimated from the densities of Cs-134 and Cs-17 deposited on the ground or I-129 with a long half-life period.

However, as shown in Section II-2, it cannot be maintained that the dose rate information consistently corresponded to the time history of the I-131 concentration in the plume. In addition, the deposition on the ground surface is an integral value of the deposition caused by the plumes that arrived, and the deposition conditions varied significantly depending on the surrounding environment. Therefore, there are limitations to the estimation of the time history based on such data. By applying this method to the data of the monitoring posts in various locations where a $\mathrm{NaI}(\mathrm{Tl})$ scintillation detector was used, including the monitoring posts in Fukushima Prefecture for which the measurement data have already been disclosed, there is a possibility that the time history of the I-131 concentration in the plumes in various areas during the early stages of the accident that occurred at Fukushima Daiichi Nuclear Power Plant can be obtained, although the areas are limited to those near the monitoring posts. It is considered that the time history of the I-131 concentration in the plumes at the monitoring posts is useful in improving the accuracy of estimation from atmospheric dispersion models and also enables a more accurate evaluation of the equivalent dose to which the thyroid gland is exposed through linking this information with behavioral data.

\section{References}

1) H. Hirayama, H. Matumura, Y. Namito, T. Sanami, "Estimation of radionuclide concentration in plume using pulse height distribution measured by $\mathrm{LaBr}_{3}$ scintillation detector and its response to radionuclides in plume calculated with egs5," Trans. At. Energy Soc. Jpn., 12, 304-310 (2012). [in Japanese]

2) T. Ohkura, T. Oishi, M. Taki, Y. Shibanuma, M. Kikuchi, H. Akino, Y. Kikuta, M, Kawasaki, J. Saegusa, M. Tsutsumi, H. Ogose, S. Tamura, T. Sawahata, Emergency Monitoring of Environment Radiation and Atmospheric Radionuclides at Nuclear Science Research Institutes, JAEA Following the Accident of Fukushima Daiichi Nuclear Power Plant, JAEA-DATA/Code 2012-010 (2012).

3) Y. Namito, H. Nakamura, A. Toyoda, K. Iijima, H. Iwase, S. Ban, H. Hirayama, "Transformation of a system consisting of plane isotropic source and unit sphere detector into a system consisting of point isotropic source and plane detector in Monte Carlo calculation." J. Nucl. Sci. Technol., 49. 167-172 (2012).

4) H. Hirayama,Y. Namito, A. F. Bielajew, S. J. Wilderman, W. R. Nelson. The EGS5 Code System. SLAC-R-730 (2005) and KEK Report 2005-8 (2005). 